\title{
MODELOS EXPLICATIVOS PARA O USO DE ÁLCOOL E DROGAS E A PRÁTICA DA ENFERMAGEM
}

\author{
Sandra Cristina Pillon ${ }^{1}$ \\ Margarita Antonia Villar Luis ${ }^{2}$
}

Pillon SC, Luis MAV. Modelos explicativos para o uso de álcool e drogas e a prática da enfermagem. Rev Latino-am Enfermagem 2004 julho-agosto; 12(4):676-82.

A prática da assistência, pesquisa e ensino em enfermagem tem proporcionado o acúmulo de considerável experiência sobre o cuidado ao paciente com problemas relacionados ao uso do álcool. Essa experiência tem mostrado que existe entre os profissionais de enfermagem em geral grande necessidade de aquisição de conhecimentos teóricos que propiciem a revisão dos modelos ou protocolos informais que embasam a prática nessa área. Este artigo tem como objetivo realizar breve revisão de literatura, que visa apresentar registro dos principais modelos teóricos existentes na literatura especializada explicativo do fenômeno do uso do álcool e drogas. Concluímos que, através do conhecimento dos modelos explicativos, o enfermeiro pode buscar meios que facilitem sua prática de acordo com as necessidades da demanda de saúde.

DESCRITORES: alcoolismo; enfermagem

\section{EXPLANATORY MODELS FOR ALCOHOL AND DRUGS USE AND THE NURSING PRACTICE}

Nursing care practice, research and teaching have led to the accumulation of considerable experience in care for patients with problems related to alcohol abuse. This experience has shown that, among nursing professionals in general, there is a great need to acquire theoretical knowledge, to review the models or informal protocols that support practice in this area. This article aims at examining literature on the main theoretical models to explain alcohol and drugs abuse. It is concluded that, through an understanding of the explanatory models, nurses can seek means of facilitating their practice according to the health demand needs.

DESCRIPTORS: alcoholism; nursing

\section{MODELOS EXPLICATIVOS DEL USO DE ALCOHOL Y DROGAS Y LAS PRÁCTICAS EN ENFERMERÍA}

La práctica asistencial, investigación y educación en enfermería han proporcionado una experiencia considerable en el cuidado al paciente con problemas relacionados al uso de alcohol. Esta experiencia mostró que existe entre los profesionales de enfermería en general una gran necesidad de adquirir conocimientos teóricos que los posibiliten revisar los modelos o protocolos informales en que basan su actuación práctica en este área. La finalidad de este artículo es hacer una revisión de la literatura sobre los principales modelos teóricos existentes para explicar el fenómeno del uso de alcohol y drogas. Concluímos que, a través del conocimiento de los modelos explicativos, el enfermero puede buscar medios que faciliten su práctica de acuerdo con las necesidades de la demanda de salud.

DESCRIPTORES: alcoholismo; enfermería

\footnotetext{
${ }^{1}$ Professor Doutor, e-mail: pillon@eerp.usp.br; ${ }^{2}$ Professor Titular. Escola de Enfermagem de Ribeirão Preto, da Universidade de São Paulo, Centro Colaborador da OMS para o desenvolvimento da pesquisa em enfermagem
} 
INTRODUÇÃO

$\boldsymbol{A}$ literatura sobre a dependência de álcool no Brasil, embora seja escassa e com as limitações decorrentes do uso das mais variadas metodologias pelos pesquisadores, tem mostrado uma prevalência variando de 3 a $10 \%$ na população geral adulta $^{(1)}$. Recente levantamento domiciliar, realizado pelo CEBRID, no ano de 1999, em 24 cidades do Estado de São Paulo, com mais de 200 mil habitantes, mostrou dados identificando consumo de álcool e cigarro semelhante ao dos EUA, e um número estimado de dependentes de 6,6\% para ambas as substâncias, sendo ambas drogas particularmente incidentes entre homens de 18 a 24 anos $^{(2)}$.

Entre as drogas psicotrópicas, o álcool parece ser a substância mais consumida no Brasil. A maioria dos estudos de prevalência tem sido feito em populações que buscam assistência médica. As taxas de prevalência de abuso de álcool nessa população variam de 20 a $50 \%{ }^{(3-}$ 5).

Segundo a Secretaria de Saúde, o alcoolismo é responsável por $50 \%$ das internações psiquiátricas masculinas, $20 \%$ em unidade de gastroenterologia ${ }^{(6)} \mathrm{e}$ $90 \%$ das internações por dependência de drogas em hospitais psiquiátricos brasileiros ${ }^{(7-8)}$. Pessoas com problemas relacionados ao álcool são hospitalizados quatro vezes mais que a população em geral ${ }^{(9)}$.

As mudanças nas necessidades de saúde da população e a própria história do uso de álcool e outras drogas têm solicitado a existência da demanda de novos serviços. Para oferecer centros de atendimento à saúde em geral (públicos e privados) compatíveis com essa realidade, o enfermeiro, dentro das suas funções, deve estar apto a absorver tais mudanças. Recentemente o Ministério da Saúde baixou a Portaria n` $816 / \mathrm{GM}$, regulando o atendimento do dependente de drogas e álcool em Centros de Atenção Psicossocial (CAPS-AD) ${ }^{(10)}$, prevendo uma equipe mínima da qual os enfermeiros e auxiliares de enfermagem fazem parte. Se, antes da legislação, a vivência prática já solicitava os conhecimentos específicos, a exigência da inclusão do enfermeiro na equipe de saúde que presta cuidados aos dependentes consolidou essa necessidade em todo o País. Por outro lado, o enfermeiro tem potencialidade à demanda, explorarando alternativas, fazendo as adaptações necessárias nos seus planos assistenciais em geral e promovendo a assistência aos pacientes com problemas decorrentes do consumo de álcool e drogas ${ }^{(11)}$.

A experiência em outros países tem mostrado que os novos modelos assistenciais disponíveis à enfermagem, nessa área, tem como objetivo promover iniciativas no âmbito comunitário bem como na assistência direta tanto ao indivíduo, quanto à família e grupos específicos da comunidade $^{(12)}$.

Conforme exposto, os estudos realizados durante a última década mostram que a prevalência do uso e abuso de álcool se manteve alta, gerando graves problemas sociais e de saúde e a magnitude desses problemas é evidente. Embora consistente, o progresso tem sido lento nos componentes da educação sobre álcool que são considerados essenciais na formação profissional do enfermeiro. Esses componentes incluem: 1- conhecimento das atitudes frente ao usuário e aos problemas relacionados, 2- obtenção de educação formal sobre o tema, 3- mudanças de atitudes.

Considerando a necessidade de ampliar e divulgar conhecimentos teóricos relacionados à dependência química, o presente artigo visa apresentar registro dos principais modelos teóricos explicativos existentes para o fenômeno do uso do álcool e outras drogas, bem como algumas estratégias norteadoras para a prática da enfermagem junto ao usuário.

\section{MODELOS EXPLICATIVOS AO FENÔMENO DO USO DO ÁLCOOL E DROGAS}

Ao longo do tempo, os autores especialistas ${ }^{(11,13-}$ 14) desenvolveram diversos modelos referentes ao entendimento das dimensões do problema do consumo de álcool e drogas. Cada enfoque se concentra exclusivamente em um subconjunto ou aspecto dos fenômenos gerais. Tendo como base seus registros, elaborou-se uma síntese dos modelos mais utilizados no tratamento e ou prevenção, contendo sua concepção do problema e principais pressupostos, os quais são apresentados na seqüência:

\section{MODELO ÉTICO LEGAL}

Trata-se de concepção vinculada aos profissionais de direito e segurança social. Segundo as proposições teóricas desse enfoque, as causas estão nas atitudes 
anti-sociais e/ou imorais de certos grupos de transgressores. Nesse modelo, considera-se o problema como basicamente qualquer ato de transgressão que requer interferência baseada em sua sanção legal; não há aprofundamento da análise da dependência da substância e dos processos que a determinam. As drogas e seu consumo são importantes e requerem a atenção na medida em que se apresentam como causadores de danos graves aos indivíduos e à sociedade. A intervenção preventiva se apóia em medidas de caráter positivo e punitivo, operando por meios dos sistemas legislativo, judicial e policial.

\section{MODELO MORAL}

Neste modelo, os indivíduos são considerados responsáveis tanto pelo início e o desenvolvimento do problema, quanto pelas soluções e acredita-se que necessitam apenas de motivação apropriada. A principal limitação do modelo moral é que as pessoas são levadas a sentir-se culpadas pelo desenvolvimento do problema e a pensar que, de alguma forma, Ihes faltam força de vontade ou "fibra moral", por não conseguirem alterar com sucesso seu comportamento.

\section{MODELO MÉDICO OU DE DOENÇA}

O modelo médico de dependência baseia-se na suposição de que a dependência de substâncias psicoativas seja entidade nosológica distinta e de caráter progressivo, com origens ou manifestações físicas e necessita de tratamento médico. Diversas entidades têm se empenhado em que seja utilizado o termo "dependência em detrimento de outros com maior conotação moral" (vício) e que esse problema seja visto como um conjunto de sintomas clínicos.

O conceito do alcoolismo como doença foi proposto inicialmente por Jellinek, em 1960. Nesse modelo, o comportamento do uso da substância é visto como progressivo, incurável e a causa da doença está relacionada aos fatores genéticos, biológicos, estruturais de natureza química, tal como tem mostrado alguns estudos $^{(15)}$. Existem fortes evidências de que o início do alcoolismo está geneticamente predeterminado no individuo ${ }^{(16)}$. Contudo, a predisposição genética pode também proteger alguns indivíduos que têm metabolismo genético sensível para tolerar o uso de substâncias psicoativas tais como o álcool ${ }^{(17)}$.

Esse modelo também implica em aceitar que o uso da substância exerce papel de doença e o indivíduo espera ser tratado como doente. O enfoque do tratamento implica na recuperação do uso, abuso e/ou dependência da substância, sendo a abstinência total objetivo a ser alcançado. Como exemplo, os grupos de auto-ajuda (AA Alcoólatras Anônimos).

A intervenção preventiva, nesse modelo, centrase no valor que as pessoas dão à saúde, e fundamenta a educação no conhecimento da ação e prejuízos de determinadas drogas sobre o organismo, bem como as modificações que provocam sobre o comportamento e atitudes das pessoas. Nesse modelo considera-se que 0 indivíduo conscientizado pela educação recebida evita o uso abusivo.

\section{MODELO PSICOLÓGICO OU PSICOSSOCIAL}

Este modelo inclui o aprendizado social, a interação familiar e os traços da personalidade do indivíduo. O modelo de aprendizado social propõe que o comportamento social é aprendido através da observação e imitação. Isto é, mostra que o exemplo dos pais é um importante fator no padrão inicial do consumo de substâncias, especialmente naquelas pessoas com habilidades sociais precárias. O uso do álcool é socialmente adquirido, os padrões de comportamento aprendidos são mantidos por fatores cognitivos, pela influência de modelos, expectativas e indicadores, e pela interação do comportamento com reforços do meio, incluindo também os fatores genéticos ${ }^{(18)}$.

O modelo da interação familiar enfatiza a função do comportamento dos pais no uso da bebida no caso do alcoolismo. O ambiente familiar é visto como parte importante na determinação do consumo do álcool e sugere que o alcoolismo está consistentemente associado com negligência, distanciamento emocional, rejeição dos pais e tensão familiar.

A atribuição de características específicas de personalidade, no caso do uso de substâncias, estaria associada a muitos fatores, dentre eles a falta de maturidade, conflitos intrapessoais e interpessoais, baixa auto-estima, ou ter como base problemas psiquiátricos como depressão ou transtorno de ansiedade, dentre outros. 
MODELO

SOCIOLÓGICO

\section{SOCIOCULTURAL}

Este modelo concebe a problemática das drogas como resultado de um número de forças sociais. A sua explicação a respeito do uso de substâncias enfatiza a função do meio cultural com suas crenças, valores e atitudes que conduzem a comunidade ou seus grupos específicos no caminho da abstenção ou do uso de drogas. É um modelo ambientalista, que destaca a importância do ambiente na conduta do indivíduo; na interação de elementos sociológicos (do grupo ou subcultura à qual ele pertence) e culturais (costumes e tradições). Para esse modelo, atitudes culturais com relação ao uso de droga psicoativa podem exercer função importante na determinação do comportamento individual. Em algumas culturas, a abstinência pode ser regra e em outras o uso pode ser parte de um ritual religioso e cerimonial ou aceito como droga social ou recreacional. Ainda no contexto desse modelo, os fatores sociológicos tais como o desemprego, a privação social, e outros, podem ter efeitos importantes sobre o início e o uso continuado da substância pelo indivíduo. Também o sexo, idade, religião, etnicidade, condições socioeconômicas e ambiente familiar influenciam diretamente na opção individual de usar ou não a substância.

A ação preventiva proposta por esse modelo tem como objetivo fundamental a mudança nas condições sociais dirigidas a grupos marginalizados, visando a melhora da qualidade de vida e sua integração na comunidade.

Para melhor visualizar os pressupostos centrais e as características desses modelos foi elaborada a Tabela 1, que permite identificar a evolução das maneiras de explicar o fenômeno da adição.

Tabela 1 - Apresentação das multifaces dos modelos explicativos para o uso de substâncias psicoativas ${ }^{(11)}$.

\begin{tabular}{|c|c|c|c|c|}
\hline & Moral & Doença & Comportamento & Psicossocial \\
\hline Etiologia & Fraqueza de caráter & $\begin{array}{l}\text { Fatores biológicos } \\
\text { possivelmente } \\
\text { genéticos }\end{array}$ & $\begin{array}{l}\text { Distúrbio do } \\
\text { comportamento e do } \\
\text { aprendizado }\end{array}$ & $\begin{array}{c}\text { Fatores ambientais e } \\
\text { sociais }\end{array}$ \\
\hline $\begin{array}{l}\text { Objetivo do } \\
\text { Tratam ento }\end{array}$ & $\begin{array}{c}\text { Controle do } \\
\text { comportam ento } \\
\text { através de } \\
\text { desencorajamento e } \\
\text { punição }\end{array}$ & $\begin{array}{c}\text { Abstinência para } \\
\text { interromper a } \\
\text { progressão da } \\
\text { doença }\end{array}$ & $\begin{array}{c}\text { Alternativa do } \\
\text { comportamento e do } \\
\text { aprendizado ou } \\
\text { incompatibilidade com o } \\
\text { uso da substância }\end{array}$ & $\begin{array}{c}\text { Melhora o } \\
\text { funcionamento social }\end{array}$ \\
\hline Vantagens & $\begin{array}{l}\text { Responsabilidade } \\
\text { para mudar o uso } \\
\text { escondido }\end{array}$ & $\begin{array}{l}\text { Sem culpa ou } \\
\text { punição }\end{array}$ & $\begin{array}{l}\text { Sem culpa ou punição. } \\
\text { O usuário é responsável } \\
\text { por novos aprendizados }\end{array}$ & $\begin{array}{c}\text { Fácil integração com } \\
\text { outros modelos }\end{array}$ \\
\hline Desvantagens & Punição & $\begin{array}{c}\text { O usuário é } \\
\text { isento da } \\
\text { responsabilidade nas } \\
\text { mudanças. Ignora os } \\
\text { fatores psicológicos, } \\
\text { culturais e } \\
\text { ambientais }\end{array}$ & $\begin{array}{l}\text { A tendência a ignorar } \\
\text { que o uso excessivo da } \\
\text { substância afeta a } \\
\text { personalidade do } \\
\text { indivíduo }\end{array}$ & $\begin{array}{c}\text { Mudanças nas } \\
\text { situações sociais são } \\
\text { suficientes para a } \\
\text { recuperação }\end{array}$ \\
\hline
\end{tabular}

É importante lembrar que tais modelos se superpõem na prática e que não existe um modelo correto para os problemas relacionados ao álcool, assim como existe um modelo de "aeronave" ${ }^{\text {"11) }}$. As idéias valiosas, entre a estrutura e o funcionamento, podem vir de um padrão madeira. Todos os modelos procuram explicar a origem dos problemas relacionados ao uso do álcool e outras drogas, mas enquanto uns enfocam apenas um aspecto da intervenção numa abordagem em particular, existem outros que esboçam uma variedade de opções de intervenções. Apenas se desencoraja a visão do problema enquanto questão relativa ao âmbito da moral (indivíduo mau-caráter). Isso não significa a ausência desse julgamento por parte da população geral e inclusive entre os profissionais. Fato esse, por vezes, constatado no atendimento aos usuários de substâncias. Tendo uma amplitude de ação maior, essa apresentação teve o propósito de oferecer diferentes perspectivas no entendimento do uso das substâncias psicoativas pelas diferentes áreas de conhecimento para subsidiar propostas de atuação. O uso da substância e o comportamento humano são questões complexas que requerem enfoque holístico em ambos os focos: entendimento da causa do uso e sua aplicação no atendimento. 
A PRÁTICA DA ENFERMAGEM AOS USUÁRIOS OU DEPENDENTES DE ÁLCOOL

Não existe apenas um modelo a seguir para o planejamento de cuidados da enfermagem na área da dependência química. Essa prática muitas vezes tem emergido, alternada e direcionada, de acordo com as necessidades de repostas aos problemas de saúde das populações, pois está diretamente ligada ao sistema de saúde e centrada nos cuidados gerais de saúde. Por isso mesmo, nem sempre tem atendido as especificidades dos usuários de substâncias psicoativas.

Há de se salientar que os modelos são tentativas teóricas para explicar um fenômeno complexo e multifacetado que é o uso de álcool e drogas. Por isso, a assistência ao usuário é uma prática complexa e, freqüentemente, o profissional pode avaliar seu cliente sob o olhar de mais de um modelo ao mesmo tempo: o moral, quando ele "não ouve" seus conselhos, o médico, quando executa procedimentos terapêuticos; o social, quando não identifica a retaguarda necessária para a reintegração social.

Os modelos explicativos para o uso do álcool e outras drogas, atualmente mais utilizados em nosso meio e que têm contribuído para modificações desse comportamento, têm se baseado no modelo médico, centralizado nas formulações da psiquiatria (hospitalização, medicalização), havendo possibilidade de abertura para o contexto da saúde pública (saúde da família e redução de danos, trabalhos comunitários).

No caso do uso de álcool, seguindo a definição ao tratamento, o Instituto de Saúde propõe que “... é uma gama de serviços que incluindo a avaliação diagnóstica, aconselhamentos e cuidados de saúde geral, psicológica e social para os usuários de substâncias cuja saúde está comprometida $^{(19)}$.

O tratamento compõe-se de dois elementos, os procedimentos terapêuticos visando prevenir a cronificação dos problemas e o processo terapêutico em si mesmo que envolve o ambiente e o contexto interpessoal em que os procedimentos são implementados para a obtenção do sucesso...". O tratamento do alcoolismo é então uma combinação de procedimentos e processos terapêuticos que interagem de forma complexa ao longo de um continuum $^{(19)}$.

Enfatizou-se o tratamento ao uso nocivo do álcool, por ser essa a substância psicoativa de uso mais freqüente.
O cuidado de enfermagem aos usuários de álcool também pode ser oferecido seguindo tais parâmetros, mantendose a idéia de continuum na sua prática, incluindo atividades terapêuticas, assim como a intervenção primária (orientações breves e objetivas), a identificação precoce através de testes curtos e padronizados (AUDIT), avaliação do estado de saúde e diagnóstico das necessidades afetadas, a prevenção de recaídas, intervenção breve, aconselhamentos com objetivo e, por exemplo, o direcionamento do usuário para a abstinência do álcool.

Os princípios básicos para a assistência aos usuários de álcool e outras drogas, não se diferenciam das demais áreas da enfermagem, há necessidade de se promover a aliança terapêutica através de um ambiente acolhedor, da empatia (fundamental para a motivação), conduzindo ao relacionamento interpessoal. Garantindo ao indivíduo assistência integral e contínua e contribuindo para a competência coletiva do trabalho da equipe. É particularmente importante boa comunicação e o trabalho cooperativo. O paciente deve ser entendido e abordado sob a ótica da totalidade numa perspectiva holística (a chave da intervenção terapêutica) que tem como foco principal o ser humano na compreensão e tratamento do problema ou desconforto. Nessa visão, o uso da substância química é visto como o agente gerador de malefícios, que precisa ser tratado de alguma maneira, mas, inegavelmente, o indivíduo deve receber os aportes necessários para alcançar o seu equilíbrio. Nesse sentido, o enfermeiro pode auxiliar nessa instrumentalização, incentivando e apoiando os usuários a assumirem a responsabilidade pela melhora na qualidade de sua vida em todos os níveis.

Já, no atendimento inicial, o enfermeiro identifica os problemas associados ao uso das substâncias, ouve as queixas do paciente, percebe os mecanismos de defesa envolvidos (negação, por exemplo), identifica o padrão de consumo da substância no dia, no mês e ao longo da história do paciente, na busca da caracterização do uso nocivo ou dependência. Com base nisso, ele pode classificar esse usuário como dependente (ou não) e de que substância. Nessa primeira entrevista, é importante 0 enfermeiro ouvir as queixas do paciente, valorizar seus problemas e não fazer julgamentos de valor. Nessa ocasião, podem ser utilizados instrumentos padronizados para a coleta de informações. Ao finalizar a entrevista inicial, é importante dar um retorno sobre as queixas do paciente e apresentar os objetivos do programa de atendimento, o 
contrato terapêutico, a necessidade de participação de um familiar no tratamento, possíveis interconsultas com outros profissionais da equipe e o planejamento do próximo atendimento.

No processo de acompanhamento, que pode ter como objetivo a desintoxicação (programa de atendimento aproximadamente de 7 a 10 dias), o enfermeiro realiza o monitoramento dos sintomas da síndrome de abstinência, prevenindo assim maiores complicações futuras. Além disso, conhecendo a história atual do uso do álcool e de outras substâncias, padrão de consumo da substância e, ciente dos problemas relacionados ao uso, pode realizar o acolhimento e breve sensibilização confrontando os problemas relatados pelo paciente e sua associação como o uso da substância, tendo com isso indicadores para avaliar o grau de motivação para o tratamento, bem como identificando a necessidade ou não de encaminhamento para avaliação médica (comorbidade e necessidade de medicalização).

Uma das estratégias que pode ser utilizada pelo enfermeiro no processo de tratamento é o aconselhamento (cuja base é a terapia - cognitiva), buscando fornecer ao paciente conselhos diretos que promovam reflexões e mudanças de comportamento de maneiras enfáticas. Algumas vezes resumem-se a orientações específicas e, em outras, são necessárias indicações como a redução do consumo e a substituição de uma substância por outra de "menor nocividade", e até sugestões mais drásticas, como a indicação de abstinência total.

Nesse contexto, o enfermeiro pode utilizar outras estratégias que são desenvolvidas concomitantemente no processo de tratamento e reabilitação do usuário de álcool e outras drogas; a prevenção da recaída, a intervenção motivacional e a intervenção breve.

A prevenção de recaída consiste num repertório de meios e estratégias que o indivíduo usuário pode utilizar para evitar recaída em certos comportamentos que fazem parte do quadro da dependência ${ }^{(20)}$. Seu fundamento remonta aos princípios da teoria do aprendizado social, constituindo um programa estruturado, visando ao autocontrole, que inclui procedimentos de treinamento de habilidades, intervenções e mudanças no estilo de vida ${ }^{(21)}$. O foco central é a manutenção do processo de mudança do comportamento, objetivando inclusive prevenir a ocorrência de lapso (uso da substância, sem continuidade), mais freqüente no início do tratamento e que esse não se transforme em recaída (retorno ao padrão de uso anterior ao tratamento) ${ }^{(20)}$.
Daí a importância da intervenção motivacional ${ }^{(22)}$, enquanto estratégia baseada em pressupostos da psicologia motivacional. O enfermeiro pode exercê-la incentivando a motivação do usuário para mudar seu comportamento de beber, planejando suas ações de maneira a se proteger das situações de risco e a fazer planos para o futuro.

A intervenção breve ${ }^{(20)}$ envolve procedimentos de ensino de meios de autocontrole para atingir os objetivos da abstinência ou diminuição da quantidade e/ou freqüência de uso da substância. Pode ser feita em sessões breves por meio da técnica de aconselhamento, onde o problema é avaliado recebendo retorno personalizado do enfermeiro, que procura trabalhar, nessa intervenção, os mecanismos de resistência e negação do indivíduo. É necessário o desenvolvimento de acordo mútuo por meio do qual sejam explicitados os objetivos do usuário e aqueles do tratamento, podendo ser utilizados vários meios para favorecer a adesão: manual de atividades diárias a ser preenchido pelo cliente, cartão de controle pessoal do uso da substância, contatos telefônicos, visitas domiciliares, encaminhamento a outros profissionais e parcerias com grupos de auto-ajuda.

No processo de tratamento e reabilitação do usuário de álcool ou drogas familiares e cuidadores devem ser incluídos, o enfermeiro pode incentivar a participação em entrevistas individuais e em grupos de apoio para orientação e acolhimento do cliente, pois podem ser de importância fundamental no auxílio às mudanças de comportamento do usuário, necessárias para o desenvolvimento de um estilo de vida mais saudável.

\section{CONSIDERAÇÕES}

O uso da substância e o comportamento humano são questões complexas que requerem enfoque holístico de modo contínuo em ambos os focos: entendimento da causa do uso e sua aplicação da prática assistencial. Os modelos procuram explicar a origem dos problemas relacionados ao álcool e drogas, mas enquanto uns enfocam o problema numa abordagem em particular, existem outros que esboçam variedade de opções para intervenções. Tendo amplitude de ação maior essa apresentação teve o propósito de oferecer diferentes perspectivas no entendimento do uso das substâncias pelas diferentes áreas de conhecimento para subsidiar 
propostas de atuação de enfermagem. Nesse sentido, foram apresentadas as principais estratégias que 0 enfermeiro pode utilizar no tratamento e reabilitação dos dependentes.

O enfermeiro, desde que capacitado, pode

\section{REFERÊNCIAS BIBLIOGRÁFICAS}

1. Almeida N Filho, Mari JJ, Coutinho E, França J, Fernandes JG, Andreoli SB, et al. Estudo Multicêntrico de morbidade psiquiátrica em áreas urbanas brasileiras (Brasília, São Paulo, Porto Alegre). Rev ABP - APAL 1992; 14(3):93-104.

2. Góes MA. A ameaça da drogas legais. Pesqui FAPESP 2000 abril; 52:14-21.

3. Masur J. Conjecturas sobre o uso milenar de bebidas alcoólicas. Ci Cult 1978; 30(5):531-4.

4. Mincis M. Doença hepática alcoólica: atualização. Rev Hospital São Paulo - Escola Paulista de Medicina 1992; 4(1/ 4):23-31.

5. CEBRID - IV Levantamento sobre o uso de drogas entre estudantes de 1 e 2 grau em 10 capitais brasileiras. São Paulo: CEBRID/UNIFESP; 1997.

6. Figlie NB, Pillon SC, Laranjeira RR, Dunn J. O AUDIT identifica a necessidade de interconsulta específica para dependentes de álcool no Hospital Geral?. JBP 1997; 46(11):589-93.

7. Carlini A, Carlini-Cotrin B. A preponderante participação do álcool nas internações por dependências e por psicoses induzidas por drogas psicotrópicas no Brasil, triênio 1987 1989. JBP 1991; 40(6):291-8.

8. Noto AR, Carlini EA. Internações hospitalares provocadas por drogas: análise de sete anos consecutivos (1987-1993). Rev ABP - APAL 1995; 17(13):107-14.

9. Zook CJ, Moore FD. High cost user of medical care. N Engl J Med 1980, 302(18):996-1001.

10. Portaria 816 de 30 de abril de 2002. Dispõe Programa Nacional de Atenção Comunitária Integrada a Usuários de Álcool e Outras Drogas. Diário Oficial da União 2002 maio 03: Seção 1; 29-30.

11. Rassool GH, Gafoor M. Addiction Nursing: Perspectives on professional and clinical practice. Cheltenhan: Ed Stanley Thornes; 1997.

12. Coyne P, Clancy C. Out of sight, out of mind in Aids: The nursing response. London: Chapman \& Hall; 1996.

13. Allen K. Attitudes of registered nurses toward alcoholic patients in a general hospital population. Int J Add 1993; 28(9):923-30.

14. Manson L, Ritson B. Alcohol and Health. A hand book for nurses, midwives and health visitors. London: Medical Council on Alcoholism; 1984.

15. Vaillant GE. The natural history of alcoholism. Cambridge: Harvard University Press; 1983.

16. Cloninger CR. Neurogenetic adaptative mechanism in alcoholism. Science 1987; 236:410-5.

17. Wolf $\mathrm{P} \mathrm{H}$. Ethnic differences in alcohol sensitivity. Science 1972;175:449-50.

18. Nathan PE. Behaviour theory and behavioural theories of alcoholics. In: Galenter Recent Development in Alcoholism. New York: Plenum; 1983. contribuir efetivamente com a sua prática nessa área de grande relevância social. O conhecimento dos modelos explicativos pode fornecer meios que subsidiem sua atuação de acordo com as necessidades dos usuários e das diretrizes de saúde.

19. Institute of Medicine. Broadening the base of treatment of alcohol problems. London: National Academy of Science; 1990.

20. Marlatt A, Gordon J. Prevenção da Recaída: estratégia e manutenção no tratamento de comportamentos aditivos. Porto Alegre: Artes Médicas; 1993.

21. Beck AT, Wright F, Newmann C, Liese B. Cognitive therapy of substance abuse. New York: Guilford Press; 1993.

22. Miller WR, Rollnick S. Motivational Interviewing: preparing people to change. New York: Guilford Press; 1991. 